\title{
Giant Oocytes with Two Meiotic Spindles and Two Polar Bodies: Report of Two Cases
}

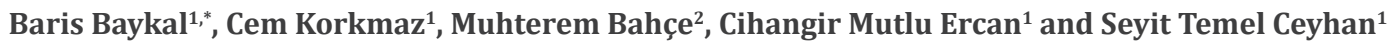 \\ ${ }^{1}$ ART Centre, Department of Obstetrics and Gynecology, Gulhane Training and Research Hospital, University of Health Sciences, Ankara, \\ Turkey. \\ ${ }^{2}$ MBGENLAB Genetic Diseases Diagnosis Center, Ankara, Turkey.
}

Received: May 08, 2017; Accepted: May 22, 2017; Published: May 31, 2017

*Corresponding author: Dr. Baris Baykal, ART Centre, Department of Obstetrics and Gynecology, Gulhane Training and Research Hospital, University of Health Sciences, Ankara, Turkey. E-mail: baris_baykal@yahoo.com

\section{Introduction}

With the advent of IVF technology, the terms normal and abnormal oocytes have been defined and one type of abnormal oocyte is the "giant oocyte". Giant oocytes are defined to have a $30 \%$ larger diameter and twice the volume of normal oocytes[1,2]. Giant oocyte is a rarery observed phenomenon among humans and embryos may develop from these oocytes [2,3].The first hypothesis for the mechanism of their formation is cytoplasmic fusion of two oogonia and the second one is the lack of cytokinesis during mitotic divisions in an oogonium [4]. Fertilization and progression of a giant oocyte is suspected to be the cause of digynic triploidy, which is defined as triploidy with two maternal and one paternal complements [5]. In this case report, we present two giant oocytes, each shown to have two meiotic spindles via visualization by polarization microscope. Because giant oocytes can develop into embryos that are morphologically normal, but genetically abnormal, an embryologist has to be aware of this phenomenon. For this reason, the scientific aim of this report is to present the polarization microscopic properties of giant oocytes and increase the awareness of such oocytes among embryologists.

Keywords: Giant oocyte; meiotic spindle; polarization microscopy

\section{Case Report}

During the ICSI procedure of two patients with the aid of polarization microscope, we encountered giant oocytes with two distinct meiotic spindles and two polar bodies.

The first patient was 26 years old and the reason for infertility was detected to be poor ovarian reserve and male factor infertility. An antagonist patch protocol was applied using a total dose of 2400 units of Follitropin-alfa (Gonal-F, Merck Serono, Germany) and a toal dose of 2400 units of human menopausal gonadotrophin (Merional, IBSA, Slovakia) for 8 days. Her husband's semen analysis report was found to be oligoasthenozoospermic (sperm concentration: 2x106/ml; ICSI was performed with motile sperm) according to World Health Organization guidelines [6]. Five oocytes were collected, three of which was at MII stage, one was at MI stage and one was at GV stage. During ICSI procedure, we observed that one of the MII oocytes was a giant oocyte with two polar bodies and a diameter of $143.75 \mu \mathrm{m}$ (Fig. 1).

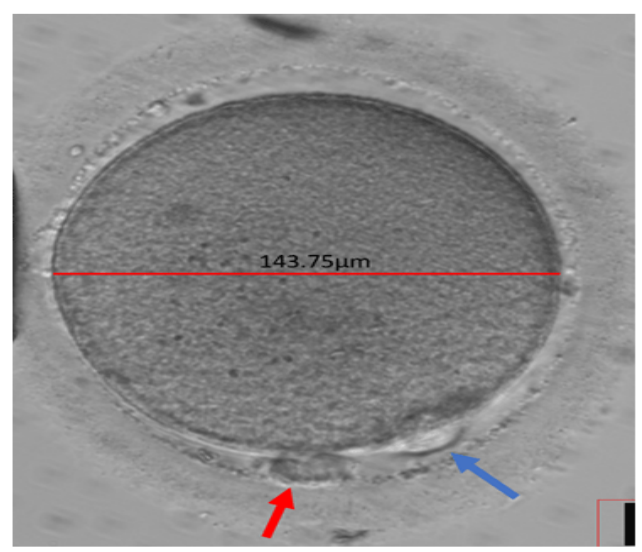

Figure 1: Giant oocyte retrieved from patient 1. It has a diameter of $146.88 \mu \mathrm{m}$ and two polar bodies (red and blue arrows).

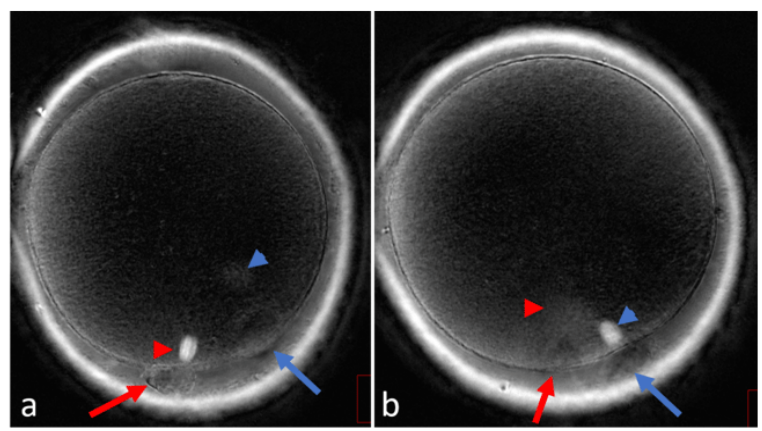

Figure 2: Polarization micrographs of the giant oocyte retrieved from patient 1. a. First polar body and the first meiotic spindle is shown by red arrow and red arrow head. The second polar body and the second meiotic spindle are marked by blue arrow and blue arrowhead. b. The positions of the polar bodies and meiotic spindles after rotating the oocyte. 
Visualization with polarization microscope (Oosight Meta Imaging System, Cambridge Research and Instrumentation, USA) revealed two meiotic spindles (Fig. 2). ICSI was performed on this oocyte. It had 3PN view on day one, 5 blastomeres on day two and 10 blastomeres on day 3 (Fig. 3). Blastomere biopsy was performed and PGD using FISH method with 7 probes (for chromosomes X, Y, 13, 14, 16, 18 and 21, revealed triploidy (Fig. 4).
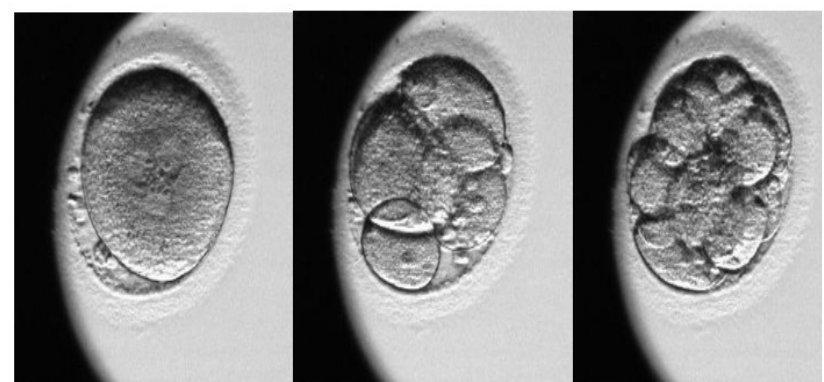

Figure 3: Progression of the giant oocyte of patient 1. a. Day 1; 3PN view. b. Day 2; the embryo has 5 blastomeres. c. Day 3; the embryo has 10 blastomeres.

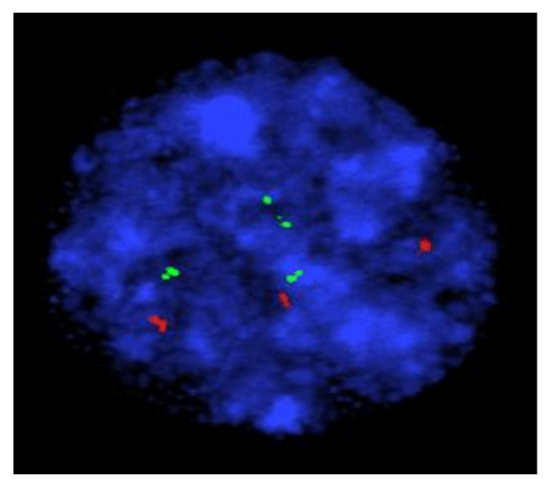

Figure 4: Fluorescent micrographs of the embryo that originated from the giant oocyte of patient 1 . Green signals refer to chromosome 14 and red signals refer to chromosome 16 .

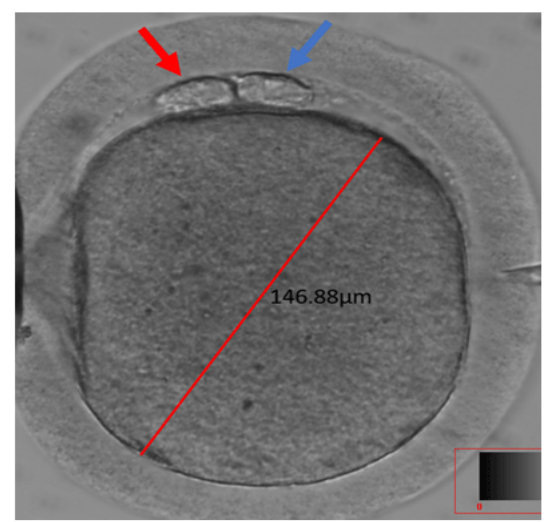

Figure 5: The giant oocyte retrieved from patient 2. Arrows point to the two polar bodies.
The other patient was 31 years old and she had endometriosis. An ultralong protocol was applied. Her husband's semen analysis report was found to be normozoospermic (sperm concentration: 100x106/ml; progressive motility: 75\%) according to World Health Organization guidelines [6]. Sixteen oocytes (15 MII and 1 MI stage) were collected. During ICSI procedure a giant oocyte with two polar bodies was encountered (Fig 5). Visualization with polarization microscope revealed two meiotic spindles both at telophase I stage (Fig 6). ICSI was performed, but no progression was observed.

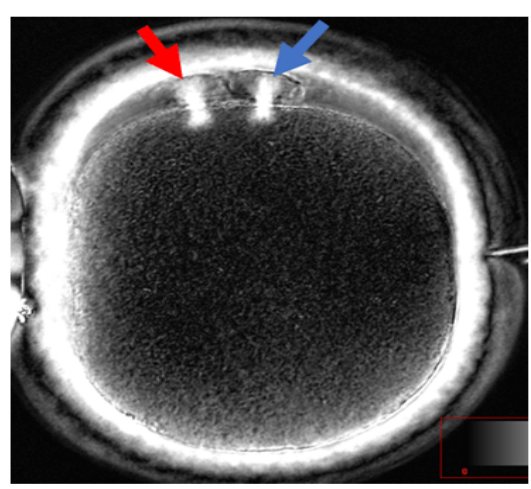

Figure 6: Polarization view of the giant oocyte from patient 2. The two spindles are at telophase I stage (arrows).

\section{Discussıon}

Our case report and the studies by other researchers prove that giant oocytes at different stages of maturation can be retrieved in human IVF cycles. The giant oocyte of patient 2 in our case also demostrates that even being evaluated as a MII stage oocyte under phase contrast optics, an oocyte can still be determined to be immature (telophase I stage) under polarization optics.

Giant oocytes can become fertilized and as reported by Rosenbusch et al., they result in triploid zygotes after monospermic fertilization even if they have 2 PN on day 1 [2]. The giant oocyte of patient 1 in our case report got fertilized with 3 PN and developed into a 10 blastomere embryo on day 3. PGD revealed triploidy.

The diameters of the giant oocytes in our cases were 146.88 $\mu \mathrm{m}$ and $143.75 \mu \mathrm{m}$ when measured without zona pellucida. Balakier et al. have reported the mean diameter of giant human oocytes as $147.73 \mu \mathrm{m}$ when measured without zona pellucida [1].

Mahadevan et al. are the first to report the recovery of a binucleated oocyte after ovarian stimulation. They proposed its formation by fusion of two adjacent oocytes [7]. Because they did not specify the size of the oocyte, we can not be sure whether it was a giant oocyte. Rosenbusch et al. have reported that all binucleate GV stage oocytes in their IVF programme have matured into giant oocytes. They have also stated that they constantly detected diploidy in giant oocytes [2]. A morphologically giant oocyte, which had two chromosome sets each accompanied by its own polar body chromosomes has been described by Angell et al. [8]. Our polarization micrograph of the giant oocyte of patient 2 also verifies this finding. 


\section{Conclusion}

Giant oocytes are rarely observed in humans. They can get fertilized and develop into morphologically high quality embryos. The findings in this case report reveal that embryos derived from giant oocytes may be triploidic. Visualization with polarization microscope reveals two meiotic spindles. Based on the findings in this case reports, transfer of the embryos derived from giant oocytes should only be considered after PGD.

\section{References}

1. Balakier H, Bouman D, Sojecki A, Librach c, Squire JA. Morphological and cytogenetic analysis of human giant oocytes and giant embryos. Hum Reprod. 2002;17(9):2394-2401.

2. Rosebush B, et al., Cytogenetic analysis of giant oocytes and zygotes to assess their relevance for the development of digynic triploidy. Hum Reprod. 2002;17(9):2388-2393.
3. Munné S, Alikani M, Cohen J. Monospermic polyploidy and atypical embryo morphology. Human Reproduction. 1994;9(3):506-510.

4. Austin CR. Anomalies of fertilization leading to triploidy. Journal of Cellular and Comparative Physiology. 1960;56(S1):1-15.

5. Hasegawa T, Harada N, Ikeda K, Ishii T, Tanaka M, et al., Digynic triploid infant surviving for 46 days. American Journal of Medical Genetics. 1999;87(4):306-310.

6. Organization WH. WHO laboratory manual for the examination and processing of human semen 2010. WHO Press: Switzerland. 2010.

7. Mahadevan MM, Fleetham MM, Taylor MM, Long-Simpson MM, Hildebrand MM. Recovery of a preovulatory binucleate oocyte in a patient following induction of ovulation for in vitro fertilization. Journal of In Vitro Fertilization and Embryo Transfer. 1988;5(5):299300 .

8. Angell RR, Xian J, Keith J. Chromosome anomalies in human oocytes in relation to age. Human Reproduction. 1993;8(7):1047-1054. Doi: 10.1093/oxfordjournals.humrep.a138190 